\title{
Microfinanciamiento para la vivienda progresiva en México: evaluación de los efectos sociales de Patrimonio Hoy en Zinacantepec, Estado de México
}

\section{Micro-financing for progressive housing in Mexico: evaluation of the social effects of Patrimonio Hoy in Zinacantepec, State of Mexico}

\author{
José DE Jesús Salazar-Cantư* \\ BRYAN W. HUSTED** \\ ESTHELA GutiÉRreZ-GARZA*** \\ KARIM ACUÑA-AsKar ${ }^{* * *}$
}

\begin{abstract}
By means of the statistical comparison of wellbeing characteristics for two groups of households in the municipality of Zinacantepec, State of Mexico, in this work we analyze the reach of the social objectives of a social responsibility program managed by the enterprise Cementos Mexicanos (Cemex). The results establish that, on the one side the users of this program build larger houses, with more rooms and faster than their non-participant neighbors, and on the other, some social objectives of the program have not been reached yet.
\end{abstract}

Keywords: social responsibility of the company, development, housing self-construction, urban zone.

\section{Resumen}

Mediante la comparación estadística de características de bienestar para dos grupos de hogares del municipio de Zinacantepec, Estado de México, en este trabajo se estima el alcance de los objetivos sociales de un programa de responsabilidad social administrado por la empresa Cementos Mexicanos (Cemex). Los resultados establecen que, por una parte, los usuarios de este programa construyen viviendas más grandes, con más habitaciones y en menos tiempo que sus vecinos no participantes, y por otra, que algunos objetivos sociales del programa aún no han sido alcanzados.

Palabras clave: responsabilidad social de la empresa, desarrollo, autoconstrucción de vivienda, zona urbana.

* Instituto Tecnológico y de Estudios Superiores de Monterrey, Campus Monterrey, México. Correo-e.: jsalazar@itesm.mx.

** Universidad de York, Toronto, Canadá. Correo-e.: bhusted@schulich.yorku.ca.

*** Universidad Autónoma de Nuevo León, México. Correos-e.: egutierr@ccr.dsi.uanl.mx; karaskar@yahoo.com. 


\section{Introducción $^{1}$}

Lograr las metas de desarrollo del milenio parte de la premisa de una mayor coparticipación entre los sectores público, privado y sociedad civil. Por su parte, el Banco Mundial, al plantear los posibles caminos para un desarrollo mundial sustentable, establece que es indispensable incluir los criterios sociales y ambientales en las decisiones de inversión de los agentes privados (Banco Mundial, 2002a: 183). Asimismo, esta institución dice que "El sector privado puede ayudar mediante la construcción de un marco que provea incentivos significativos y apropiados para que las firmas lleven adelante simultáneamente objetivos económicos, ambientales y sociales" (Banco Mundial, 2002b: 27).

Desafortunadamente, la participación del sector privado está limitada, entre otras razones, por la poca capacidad para medir los efectos sociales de proyectos privados. Diversos estudios ilustran que en México y en algunos países latinoamericanos todavía son pocas las empresas que miden el efecto derivado de sus intervenciones en acciones de responsabilidad social sobre su desempeño competitivo; por otra parte, la práctica de evaluar el efecto social de estos gastos es aún menor, casi nula (Lara, 2000; Peinado, 2004; Correa et al., 2004; Salazar, 2004; Husted y Salazar, 2005).

Cunningham y Ricks (2004) sugieren que los donantes no están interesados en medir y evaluar el desempeño de los programas sociales que apoyan. Estos autores encuentran que, contrario a lo esperado, son los propios administradores de los programas sociales quienes ven que la evaluación es una herramienta útil, ya que no sólo les permite corregir posibles fallas, sino dimensionar mejor su contribución y contar con más confianza y argumentos a la hora de presentarse a solicitar fondos y negociar contratos. Los autores señalan que la escasez tanto de medición como de evaluación de los efectos sociales más bien es producto de falta de fondos, escaso conocimiento de metodologías de evaluación y, principalmente, de su alto costo de oportunidad, expresado en acciones sociales que no tomarían lugar y tendrían que dejar de realizarse, para sufragar los gastos propios de cualquier evaluación.

La presente investigación muestra una estrategia de negocio privado que, a la vez que coadyuva a crear patrimonio habitacional en zonas de bajos ingresos, representa posibilidades claras de ganancias para la propia empresa, sin comprometer los recursos públicos que en muchos países, y en particular en México, cada vez enfrentan mayor demanda para ser invertidos en múltiples necesidades sociales alternativas.

\footnotetext{
${ }^{1}$ Este trabajo forma parte de una tesis inscrita en el programa de doctorado en ciencias sociales, con orientación en desarrollo sustentable, de la Universidad Autónoma de Nuevo León.
} 
Este análisis aborda la evaluación de efectos sociales de un programa de responsabilidad social denominado Patrimonio Hoy $(\mathrm{PH})$, administrado por Cementos Mexicanos, s.A. de c.v. (Cemex), empresa mexicana dedicada a la producción y comercialización de cemento que, por su tamaño, ocupa el segundo lugar mundial en esta industria.

Dado que PH opera en diferentes municipios de la mayoría de entidades federativas de México, y que en cada uno de ellos ha iniciado operaciones en diferentes fechas, se decidió elegir un municipio que no había sido estudiado en cuanto a los efectos sociales de $\mathrm{PH},{ }^{2}$ pero que al mismo tiempo ya contaba con casi cuatro ańos de estar en operación al momento de realizar el estudio. Este tiempo permitía ya contar con grupos de socios que habían transitado por las diferentes etapas del programa y, por tanto, se prestaba para estudiar los cambios generados por el mismo. Igualmente, Zinacantepec es un municipio representativo del tipo de lugares donde se ha llevado a cabo el programa, el cual se enfoca en zonas urbanas de ingresos bajos, por lo que los resultados para este caso pueden sugerir lo que quizá esté ocurriendo también en el resto, si bien esto no descarta la necesidad de realizar evaluaciones en otros estados más para aumentar el grado de confianza de los resultados.

La evaluación tiene dos objetivos: primero, ofrecer mayores elementos de decisión a los administradores de pH y con ello coadyuvar a que este programa alcance cada vez mejores resultados para sus destinatarios, y segundo, dimensionar los efectos sociales como una herramienta para transparentar los resultados del programa y como posible alternativa de política para gobiernos preocupados por el mejor y más eficiente uso de los recursos.

La comparación mediante pruebas estadísticas entre un grupo de control y el grupo de socios de $\mathrm{PH}$, considerando variables que representan los objetivos sociales del programa, permitió observar, entre los resultados más importantes, que hay una influencia directa de $\mathrm{PH}$ en el incremento patrimonial habitacional de los socios, a la vez que una escasa influencia sobre otros objetivos sociales, como el mejoramiento de la salud, el aumento en el ahorro o en una mayor participación en grupos comunitarios, que también persigue este programa.

${ }^{2}$ Los primeros socios de $\mathrm{PH}$ se encuentran en barrios de la periferia de Guadalajara, Jalisco. Herbst (2002) realizó un interesante análisis de los efectos de este programa en dicho lugar. Asimismo, Sandoval (2005a) publicó un reportaje con opiniones y experiencias de socios de PH en la periferia de la ciudad de México. El programa también se ha tomado como un ejemplo de los negocios basados en el concepto bottom of the pyramid (вор), base de la pirámide, que alude a las oportunidades que representan los mercados de bajos ingresos en todo el mundo (Coldewey, 2005). El 5 de octubre de 2009, Cemex recibió el Premio Hábitat que otorga la onu por dos de los programas de esta empresa orientados a brindar soluciones accesibles de vivienda, uno de ellos fue PH (El Norte, 9 de octubre, 2009). 
Este trabajo se compone de tres partes: en la primera se presenta una descripción del programa Patrimonio Hoy y de su operación en Zinacantepec; en la segunda se aborda la metodología, y en la tercera se analizan los resultados del análisis estadístico; por último se presentan las principales conclusiones.

\section{El programa Patrimonio Hoy}

\subsection{Objetivos sociales}

Patrimonio Hoy contempla los siguientes objetivos de beneficio social para sus participantes: 1) reducir el tiempo y costo de construcción de la vivienda progresiva en zonas urbanas de bajo ingreso; 2) incorporar asesoría técnica para el desarrollo de proyectos de expansión o remodelación de vivienda, y 3) aumentar el bienestar de las familias participantes en términos de ahorro familiar, valor de la vivienda, salud, lazos comunitarios y contar con una historia crediticia que les facilite obtener nuevos créditos. ${ }^{3}$

Los objetivos son claros al expresar a quién se dirige el programa y dónde se realiza, pero no mencionan en qué grado se deben lograr, cómo y cuándo; es decir, a qué porcentaje de la población objetivo se proponen llegar, cómo pretenden hacerlo y en cuánto tiempo. Así, el propio diseño de los objetivos presenta dificultades de inicio a una posible evaluación de las llamadas ex post o de resultados.

Cemex administra el programa Patrimonio Hoy como parte de sus actividades de responsabilidad social corporativa. En el sentido de Burke y Logsdon (1996), podría decirse que este programa se considera estratégico, ya que sus objetivos sociales encajan con los de la misión de la empresa. Ésta realizó una amplia investigación sobre el fenómeno de la vivienda precaria antes de comenzar el programa, y a partir de ahí configuró un diseño del mismo, adaptado a los recursos y a la forma de subsistencia de las familias de zonas urbanas de bajos ingresos. La empresa realiza alianzas con la sociedad local, sobre todo con las amas de casa, grupo especialmente vulnerable en los hogares de ingreso bajos, para promover el programa. Esta firma difunde los avances del programa por medio de su página electrónica e inscribiéndolo en concursos internacionales. Finalmente, el programa también cumple con el adjetivo de estratégico, al producir no sólo un aumento en el patrimonio familiar de sus usuarios, sino una utilidad para la empresa. Este conjunto de caracterís-

\footnotetext{
${ }^{3}$ Como seńalan Aguilar y Ander-Egg (1992: 81-82), si bien la formulación de un marco de evaluación puede partir del examen de documentos, esto no basta, es necesario preguntar a los responsables para tener una idea más acorde con la realidad. En este caso, la consulta sobre los objetivos sociales de $\mathrm{PH}$ se le hizo a Héctor Ureta, quien entonces dirigía este programa en todo el país.
} 
ticas distintivas de PH lo hacen un programa de responsabilidad social estratégica y lo alejan de manera definitiva de lo que podría ser un programa de sesgo meramente altruista o implantado bajo coerción, en el sentido de Husted y Salazar (2006).

\subsection{Justificación del programa: oportunidad de negocio y de contribución social}

En 1998 Cemex llevó a cabo una estrategia orientada a buscar mayor posicionamiento en el segmento de autoconstrucción, entre familias de bajos ingresos en México.

La cuantificación del déficit de vivienda en México no parece una tarea sencilla, existen diversas estimaciones entre las cuales hay importantes diferencias. Con datos del Censo de Población y Vivienda 2000, Kunz y Romero (2008), mediante un análisis muy detallado, calculan que la cantidad de viviendas que requerían mejoras era de 11'111,394, mientras que el número de casas nuevas necesarias era de 10'948,274.

Por su parte, Cemex ${ }^{4}$ estima que hay un déficit habitacional de cuatro millones de viviendas en México, lo cual se aproxima a los 4.2 millones calculados por la Comisión Nacional de Fomento a la Vivienda (Conafovi) (Rodríguez, 2005). Si se considera el déficit según el número de cuartos, en el año 2000 la cifra nacional alcanzaba los 8,239,752.5

Estimaciones de Damián (2005), basadas en el índice de carencia en la calidad de la vivienda, hacen ver que en México, entre los años 2000 y 2004, la pobreza en vivienda aumentó, el porcentaje de la población total con carencias habitacionales pasó de 70.5 a $74 \%$, lo que representa 77.1 millones de personas.

El Centro de Investigación y Documentación de la Casa, A.C. (CIDOc) y la Sociedad Hipotecaria Federal (sHF) (2009), estiman en 36.7 millones las personas que habitan viviendas con algún tipo de rezago y en 551,801 las viviendas con hacinamiento. Con base en estas cifras, calculan la demanda en el año 2009 de 966,063 viviendas.

Por su parte, el Consejo Nacional de Evaluación de la Política Social (Coneval), al aplicar el método de medición de la pobreza multidimensional, con datos de 2008 calculó que la población con carencias en calidad y espacios en la vivienda era de 18.62 millones de personas, mientras que aquéllos sin servicios básicos en la vivienda fue de 20.13 millones.

${ }^{4} \mathrm{http} / / /$ www.cemexmexico.com/se/se_ph.html, 26 de agosto de 2005.

${ }_{5}^{5}$ Cálculo propio con base en cifras del xiI Censo de Población y Vivienda, 2000, http://www. inegi.gob.mx/est/librerias/tabulados.asp?tabulado=tab_vi05Aa\&c=777\&e=, 7 de septiembre de 2005 . En el cálculo se consideró que un número de personas aceptable por cuarto era de dos, este criterio se tomó de Bazant (2003: 14). El procedimiento que se siguió fue calcular el déficit de cuartos al cruzar información de número de viviendas según número de ocupantes y número de cuartos. 
Si bien existen diferencias en las estimaciones, lo que queda claro es que la cantidad de demanda de vivienda en México constituye uno de los mercados potenciales más grandes, asimismo, y aún más importante, como bien señalan Kunz y Romero (2008), representa una de las carencias sociales más extendidas y de más compleja solución.

Herbst (2002) muestra que un atractivo muy importante en el mercado del cemento en zonas de bajos ingresos es la estabilidad del mismo, señala que durante las crisis económicas mexicanas, el mercado de ingresos medianos y altos ha caído en proporciones de cinco veces a una, comparado con el de ingresos bajos.

El tamaño y la estabilidad del mercado son factores muy importantes que suelen ofrecer a los dueńos y administradores de las empresas mejores horizontes de planeación y expectativas de rentabilidad. Si bien estos dos elementos de juicio atraerían a cualquier empresa, en el negocio de la construcción un punto importante de inicio es el ahorro de las familias, cualquier proyecto por pequeño que sea, les implica contar con cierto monto ahorrado y/o un financiamiento externo, este último poco o nada accesible para buena parte de la población en el sistema financiero formal, donde generalmente se exige al solicitante que sea empleado de planta (no temporal) y/o contar con garantías físicas, como bienes inmuebles. Guillermo Babatz, director general de la Sociedad Hipotecaria Federal (SHF), al referirse al otorgamiento de créditos hipotecarios señala que "no es posible atender a las familias cuyos ingresos sean menores a cinco veces el salario mínimo" (Cacho, 2005: 9). En este mismo sentido, un estudio en el ámbito nacional sobre la vivienda en zonas de bajos ingresos en México, estimó esta línea en 6.26 veces el salario mínimo (Iracheta, 2001: 72), ingreso que le alcanzaría para conseguir un crédito hipotecario, pagadero a 30 años, que le permitiría adquirir una casa de entre 30 y $33 \mathrm{~m}^{2}$ de construcción y entre 60 y $91 \mathrm{~m}^{2}$ de terreno (Iracheta, 2001: 66). Por su parte, estimaciones del Banco Mundial (2004: 220) ilustran que la población mexicana que utiliza instrumentos bancarios formales se encuentra en los deciles $7^{\circ}$ a $10^{\circ}$ de la distribución del ingreso, mientras el resto utiliza instrumentos informales o no cuenta con ninguno.

Poco se sabe acerca de las determinantes del ahorro entre las familias de bajos ingresos, así lo señala Campos, quien resalta la importancia que para las empresas representaría conocer mejor esta variable: "Cuando la teoría logre explicar cómo viven y administran sus recursos las personas de bajos recursos, los tomadores de decisiones podrán trabajar en un terreno de mayor certidumbre respecto a cómo, cuánto y cuándo intervenir" (2005: 52).

Actualmente el gobierno mexicano cuenta con diversos programas para dotar y/o mejorar la vivienda, los más importantes son administrados 
por el Fondo de la Vivienda del Instituto de Seguridad Social al Servicio de los Trabajadores del Estado (Fovissste), que apoya a los trabajadores públicos, y el Instituto del Fondo Nacional de la Vivienda para los Trabajadores (Infonavit), mismo que ofrece diferentes apoyos entre sus derechohabientes. En el caso que ocupa el presente estudio, Infonavit no parece estar representando una opción muy al alcance de la población de Zinacantepec, municipio del Estado de México, en el cual, de acuerdo con cifras del XII Censo Nacional de Población y Vivienda, ${ }^{6}$ casi la mitad de los trabajadores estaban subocupados. Por su parte, también las cifras de este censo ${ }^{7}$ mostraron que sólo $1.8 \%$ de los habitantes de Zinacantepec eran derechohabientes del IsssTE.

Otro programa público, similar al que se analiza en el presente estudio, es Ahorro y Subsidios para la Vivienda Progresiva Tu Casa, ${ }^{8}$ promovido por la Secretaría de Desarrollo Social (Sedesol), opera con fondos del Fideicomiso Fondo Nacional de Habitaciones Populares (Fonhapo). La diferencia más importante entre Tu Casa y $\mathrm{PH}$ es que el primero es un subsidio y el segundo un préstamo entre agentes privados. Tu Casa cuenta con diversas vertientes, quizá la más representativa sea la referente a las llamadas unidades básicas de vivienda, que son casas de poco menos de $21 \mathrm{~m}^{2}$ de construcción, edificadas en terrenos de $90 \mathrm{~m}^{2}$. En el año 2007 este programa contaba con un grupo estimado en poco menos de 10 millones de personas como potenciales beneficiarios directos, cuyos hogares contaban con ingresos de tres salarios mínimos o menos.

La Sedesol también cuenta con el Programa de Vivienda Rural, cuyo grupo social objetivo es distinto al de PH, ya que como se mencionó, este último opera principalmente en zonas urbanas y no en áreas rurales.

Los habitantes de Zinacantepec, al igual que muchos otros de ingresos bajos que viven en zonas urbanas y que aún no forman parte del Infonavit, cuentan con alternativas limitadas para la construcción de sus viviendas progresivas. El estudio de González (2003: 25) muestra que en muchos estados de México, el apoyo público a los procesos de autoconstrucción tradicionalmente ha sido nulo. Esta situación representa otro elemento que justifica la existencia de PH y la idea de extenderlo a lo largo de todo el país para fomentar un incremento sostenible en el número de viviendas en poblaciones de escasos recursos económicos. El mercado es amplio y la posibilidad de mejora social también, ambos elementos son fuertes determinantes para realizar inversiones de responsabilidad social estraté-

\footnotetext{
${ }^{6} \mathrm{http}: / /$ www.inegi.gob.mx/lib/olap/general_ver2/MXDQueryDatos.asp?\#Regreso\&c=5617, 5 de octubre de 2005.

${ }^{7} \mathrm{http}: / /$ www.inegi.gob.mx/est/librerias/tabulados.asp?tabulado=tab_ss01a\&c=750\&e=, $7 \mathrm{de}$ noviembre de 2005.

${ }^{8}$ http://www.sedesol.gob.mx/programas/vivah.htm, 5 de octubre de 2005. Éste sustituyó al Programa Especial de Créditos y Subsidios en la Vivienda (Prosavi).
} 
gica, que como señalan Husted y Salazar (2006), es el tipo de inversión en responsabilidad social que más aporta a la empresa y a la comunidad en el largo plazo.

\subsection{La forma en que opera $P H$}

Este programa se inauguró en barrios de la periferia de Guadalajara, Jalisco, a principios del año 2000. Por su parte, la oficina de Cemex establecida en Zinacantepec inició funciones en diciembre de 2001, y para septiembre de 2005 ya había dado servicio a casi 4,000 de los aproximadamente $26,005^{9}$ hogares de este municipio, localizado en la zona metropolitana de la ciudad de Toluca.

Cuando alguien se inscribe en el programa, el asesor de vivienda de Cemex brinda asesoría para el mejor desarrollo del proyecto. Cada participante ahorra durante dos semanas y sobre el monto ahorrado, Cemex le da un crédito de cuatro tantos, mismo que el acreditado podrá pagar durante las siguientes ocho semanas. La aportación semanal en septiembre de 2005 era de 145 pesos por semana, de los cuales 20 eran por membresía y los 125 restantes para la compra de materiales de construcción (tabique, tabicón, alambrón, alambre, cemento, mortero, arena, acabados, mobiliario doméstico, etc.). Una vez que cuenta con el crédito, el participante recibe los materiales de construcción de manera inmediata en su domicilio o bien le pueden ser almacenados hasta por un plazo de dos ańos. Los proyectos suelen durar varios ciclos de 10 semanas y, en la fecha que se realizó este estudio, representaban inversiones en materiales por 8,750 pesos distribuidos a lo largo de 70 semanas y hasta 26,250 en 210 semanas. Los participantes hacen equipos solidarios de tres personas, con un proyecto cada una, con la idea de que entre ellos se apoyen y motiven a llevar a cabo su proyecto y también a cumplir con los pagos en el tiempo acordado. En general los equipos están formados por familiares o amigos, pero también por personas que se conocieron cuando entraron al programa.

La información sobre PH la dan, vivienda por vivienda, promotoras del programa, las cuales normalmente son amas de casa que viven en la localidad y que reciben pagos en función del número de socios que logran inscribir y del cumplimiento de pago de éstos.

${ }^{9}$ Cálculo propio considerando el total de hogares en Zinacantepec, contabilizados por el INEGI en el año 2000: http://www.inegi.gob.mx/lib/olap/general_ver2/MDXQueryDatos.asp, y aplicando un factor de crecimiento igual al de la población de este municipio, proyectada por el Consejo $\mathrm{Na}$ cional de Población (Conapo) para el periodo 2000-2005 igual a 10.1\%, http://conapo.gob. $\mathrm{mx} / 00$ cifras $/ 5 . \mathrm{htm}$. El cálculo supone que el promedio de miembros en el hogar permaneció sin cambio entre 2000 y 2005, 6 de octubre de 2005. 
En 2010 el programa se encuentra operando en 22 entidades federativas de México ${ }^{10}$ y ha atendido a cerca de 200,000 familias.

A continuación se aborda la metodología empleada para determinar los efectos que ha tenido PH entre sus socios de Zinacantepec.

\section{Metodología}

Idealmente, la evaluación de efectos adopta el enfoque de dobles diferencias, en el cual se comparan, para una serie de variables de interés, los cambios suscitados en la situación de dos grupos (el de tratamiento y el de control) entre un periodo base y uno posterior. En el presente estudio no se cuenta con la imagen de los grupos en un periodo base, por lo que la comparación tomará como punto de partida información directa obtenida a partir de la aplicación de cuestionarios que llevan al agente bajo observación a comparar entre la situación actual y la previa al proyecto, para una serie de indicadores que se desprenden de los objetivos del programa, enumerados en el apartado 1.1. Esto representa una limitante del trabajo y una debilidad metodológica, que aunque es común en los trabajos de evaluación de programas sociales, se debe tratar de evitar, integrando desde el principio una caracterización completa del perfil que guarda el grupo destinatario de las ayudas y otro lo más similar posible que no las recibirá. ${ }^{11}$

A diferencia de lo que podría ser una evaluación integral del programa, ésta no observa qué tan adecuado fue el estudio previo a la implantación del mismo, su diseño, la implementación y seguimiento, la estructura y el ambiente organizacional, ni el rendimiento del personal, pues para ello sería necesaria mayor información interna de la empresa y que correspondería más al interés por la eficiencia que los propietarios de la empresa y/o los administradores pudieran tener y no de los intereses directos de los destinatarios del programa. Tampoco se intenta ver los efectos del programa en la comunidad ${ }^{12}$ (sobre el conjunto de población y el medio), que si bien pueden afectar indirectamente a los usuarios, su evaluación requeriría de mayores recursos de tiempo, monetarios y de capital humano. El análisis se centra en los efectos que el programa ha tenido en los

${ }^{10}$ El grupo de entidades se concentran en el centro y noreste del país, la información de contacto en cada entidad puede obtenerse en el mapa que aparece en la página http://www.cemexmexico.com/se/se_ph_de.html.

${ }^{11}$ Véase, por ejemplo, el informe de evaluación de Progresa (Sedesol, 2003).

${ }^{12}$ El tipo de evaluación que estudia los efectos comunitarios se conoce con el nombre de evaluación de impacto, mientras la evaluación dirigida a lo que sucedió con los destinatarios del programa se denomina evaluación de efectos (Aguilar y Ander-Egg, 1992: 131). El campo de estudio de la evaluación de efecto social (social impact assessment) es muy reciente, comprende múltiples aspectos que regularmente implican la participación de un equipo multidisciplinario (DeTombe, 2003: 287). 
participantes. Por ello aquí se habla de los objetivos sociales del programa y no de aquéllos que tienen más que ver con el desarrollo general de la zona objetivo o bien con la rentabilidad e imagen para la empresa, para los cuales diversos autores (Herbst, 2002; Flores, 2004; Sandoval, 2005a y b; Coldewey, 2005) ya han señalado los efectos positivos que este programa produce para la empresa.

\subsection{Las variables y su medición}

En el cuadro 1 se muestra la información sobre las variables empleadas en el análisis, la cual se obtuvo mediante la aplicación de un cuestionario.

\section{Variables de interés y de medición}

\begin{tabular}{|c|c|c|}
\hline $\begin{array}{l}\text { Variable de interés } \\
\text { o de control }\end{array}$ & Variable de medición & Unidades \\
\hline Ingreso familiar & $\begin{array}{l}\text { Promedio mensual de } \\
\text { ingreso agregado de los }\end{array}$ & Pesos \\
\hline Ahorro familiar & $\begin{array}{l}\text { miembros de la vivienda } \\
\text { Ahorro }\end{array}$ & $\begin{array}{l}\text { Pesos y porcentaje del total } \\
\text { de ingreso }\end{array}$ \\
\hline $\begin{array}{l}\text { Nivel escolar del jefe } \\
\text { de la vivienda }\end{array}$ & $\begin{array}{l}\text { Nivel escolar del jefe de la } \\
\text { vivienda }\end{array}$ & $\begin{array}{l}\text { 1) nula, 2) primaria, 3) } \\
\text { secundaria, 4) preparatoria, } \\
\text { 5) técnica, 6) profesional }\end{array}$ \\
\hline Tamaño de la & $\mathrm{m}^{2}$ de construcción & $\mathrm{m}^{2}$ de construcción \\
\hline vivienda & Número de cuartos & Número de cuartos \\
\hline Lazos comunitarios & $\begin{array}{l}\text { Participación en grupos } \\
\text { comunitarios }\end{array}$ & $\begin{array}{l}\text { Binaria: } \text { participa }=1, \\
\text { no }=0\end{array}$ \\
\hline Costo & $\begin{array}{l}\text { Costo de proyecto de } \\
\text { construcción (material y } \\
\text { mano de obra) }\end{array}$ & Pesos \\
\hline \multirow[t]{2}{*}{ Salud } & $\begin{array}{l}\text { Gasto promedio anual en } \\
\text { medicinas por ocupante de } \\
\text { la vivienda. }\end{array}$ & Pesos \\
\hline & $\begin{array}{l}\text { Visitas promedio anual al } \\
\text { médico por ocupante }\end{array}$ & Número de visitas \\
\hline Uso del crédito & $\begin{array}{l}\text { Cuenta con algún crédito } \\
\text { actualmente }\end{array}$ & $\begin{array}{l}\text { Cuenta con crédito }=1, \\
\text { no }=0\end{array}$ \\
\hline Expectativas & $\begin{array}{l}\text { Situación general esperada } \\
\text { por el entrevistado }\end{array}$ & $\begin{array}{l}\text { Escala de } 1 \text { a } 10 \\
\text { menos a más }\end{array}$ \\
\hline Nivel de felicidad & $\begin{array}{l}\text { Felicidad como la entiende } \\
\text { quien contesta }\end{array}$ & $\begin{array}{l}\text { Escala de } 1 \text { a } 10, \\
\text { menos a más. }\end{array}$ \\
\hline
\end{tabular}

Fuente: Elaboración propia. 


\subsection{Técnicas de análisis}

En la comparación se utilizan correlaciones, pruebas de diferencias entre medias y proporciones, diferencias en el tiempo, pruebas Chi cuadrada y análisis de regresión.

El enfoque metodológico de observación de dos grupos (tratamiento y control) permite el uso de la prueba $t$ para diferencias de medias y la prueba $z$ para diferencias de proporciones entre las variables de interés. De acuerdo con Levin (1981:360,371), en general las pruebas toman la siguiente forma:

$$
\begin{aligned}
& \mathrm{H}_{0}: \mu_{1}=\mu_{2} \\
& \mathrm{H}_{1}: \mu_{1} \neq \mu_{2},
\end{aligned}
$$

donde $\mu_{1}, \mu_{2}$ son las medias de los grupos 1 y 2 , respectivamente.

$\mathrm{Y}$ en el caso de las proporciones, la forma es:

$$
\begin{aligned}
& \mathrm{H}_{0}: p_{1}=p_{2} \\
& \mathrm{H}_{1}: p_{1} \neq p_{2}
\end{aligned}
$$

donde $p_{1}, p_{2}$ son las proporciones del grupo de interés en el total.

El modelo de análisis con un enfoque de diferencias en el tiempo para cualquier indicador I se define a continuación (Sedesol, 2003: 12).

Estimador de dobles diferencias para el cambio de los resultados en el tiempo:

$$
\mathrm{DDI}_{\mathrm{t}}=\left(\mathrm{I}_{2, \mathrm{t}}-\mathrm{I}_{1, \mathrm{t}}\right)-\left(\mathrm{I}_{2, \mathrm{t}-1}-\mathrm{I}_{1, \mathrm{t}-\mathrm{t}}\right)
$$

El presente estudio no contó con información comparativa de los grupos en el punto de partida del programa, lo cual limita el análisis de dobles diferencias a un análisis de diferencias estadísticas dentro del grupo utilizando pruebas $t$ y $z$ de medias y proporciones, respectivamente. Así, la forma posible queda expresada como:

$$
D I_{t}=\left(I_{1, t-1}-I_{1, t}\right) \text {, o bien, } D I_{t}=\left(I_{1, t}-I_{2, t}\right)
$$

En este enfoque se hacen comparaciones con lo observado por Bazant (2003) en su estudio sobre vivienda progresiva en zonas de bajos ingresos del Distrito Federal y el Estado de México, para las variables de interés.

Con la finalidad de controlar diferentes factores que pudieran tener efectos concomitantes a los de PH en las variables de interés I, se considera el uso de modelos de regresión de la siguiente forma general: 
$I_{t}=\alpha+\beta_{1} S_{t}+\beta_{2} Y_{t}+\beta_{3} E_{t}+\beta_{4} G_{t}+\beta_{5} N_{t}+\varepsilon$

donde $\mathrm{I}_{\mathrm{t}}$ es la variable de interés (objetivos sociales de $\mathrm{PH}$ ); $\mathrm{S}$ es la variable binaria (dummy) que indica pertenencia (1) o no (0) a PH; $\mathrm{Y}$ es el ingreso del hogar; E es educación del jefe de familia; $G$ es la variable binaria (dummy) que indica género del jefe de familia; $\mathrm{N}$ el número de ocupantes de la vivienda; $\alpha$ es la constante de la ecuación (resume el efecto promedio de los cambios en las variables dejadas fuera del modelo, sobre la variable dependiente); $\beta_{\mathrm{i}}$ los coeficientes relacionales entre cada una de las variables independientes y la dependiente, $i=1,2,3,4,5$, y $\varepsilon$ es el término de error del modelo.

\subsection{Muestra}

El análisis compara dos grupos: tratamiento y control. El primero de ellos es el de los socios de PH. Después de eliminar algunos casos para los cuales no se contó con información suficiente que permitiera su localización ${ }^{13} \mathrm{y}$ de restar a aquéllos que formaron parte de la prueba piloto, el tamańo del grupo fue de 62. Los socios que comenzaron el programa y lo completaron entre enero de 2002 y septiembre de 2003 fue una población menor a 100 personas, por lo que se decidió entrevistar a todos, y se dejó al resto de este grupo, menos localizable, para posibles reemplazos. Al calcular la muestra del grupo de control se consideró un muestreo estratificado para todo el municipio de Zinacantepec y se obtuvo una muestra global de 147 elementos, ${ }^{14}$ que se dividieron proporcionalmente entre las nueve áreas geoestadísticas básicas (AGEB) que abarcan el municipio de Zinacantepec.

\footnotetext{
${ }^{13}$ La prueba piloto permitió constatar que la localización de personas, cuyo domicilio o teléfono no se encuentran bien especificados en la información proporcionada por la empresa, no fue tarea fácil, en principio se esperaba que las personas que sí se localizaran pudieran dar información sobre dónde encontrar a los demás, pero en ningún caso fue así. La prueba piloto incluyó a 16 socios de PH y 16 no socios. En vista de la imposibilidad de contar con información más amplia, se decidió eliminar de la muestra a aquellas personas cuya dirección no estuviera disponible en el listado proporcionado por la empresa, en los casos excluidos abunda la descripción de "domicilio conocido, sin número" y en segundo lugar, descripciones incompletas como: "Col. Independencia, sin número", "Morelos, sin número", "Carretera a Santa María, sin número". En estricto sentido, podría decirse que el estudio versa sobre los socios concentrados en las zonas más urbanizadas de Zinacantepec.

${ }^{14}$ La fórmula aplicada es: $\mathrm{n}=\left(\mathrm{n}^{\prime}\right) /\left[1+\left(\mathrm{n}^{\prime} / \mathrm{N}\right)\right]$, donde la muestra preliminar $\mathrm{n}^{\prime}=\left(\mathrm{s}^{2} / \mathrm{V}^{2}\right)$, para la cual $s^{2}=(\mathrm{p})(\mathrm{q})$ es la varianza de la muestra $\mathrm{y} \mathrm{V}^{2}$ es la varianza de la población, igual al cuadrado del error estándar. $\mathrm{Al}$ sustituir los datos del presente caso en estas fórmulas, tenemos:
}

$$
\begin{aligned}
& s^{2}=(0.8664 \times 0.1538)=0.133252 \\
& \mathrm{~V}^{2}=(0.03) 2=0.0009 \\
& \mathrm{n}^{\prime}=(0.133252 / 0.0009)=148.0581 \\
& \mathrm{n}=(148.0581) /[1+(148.0581 / 26005)]=147.2199
\end{aligned}
$$

Las muestras particulares de cada estrato se obtienen con base en ponderaciones sobre el total que representa la población particular de cada uno de ellos. 
Los hogares a visitar en este segundo grupo fueron los vecinos más cercanos a los socios de PH. La intención principal al seguir esta orientación es poder comparar versus hogares con mayor probabilidad de tener las características socioeconómicas del socio. Este supuesto es necesario ante la inexistencia de información completa de base que permitiera distinguir, para diferentes características por hogar, cada elemento de los dos grupos bajo comparación. El número de cuestionarios a aplicar por AGEB en este segundo grupo, se dividió entre el número de socios de PH que también correspondieron a dicho AGEB. En promedio correspondieron dos cuestionarios en el grupo de control, por cada cuestionario aplicado en el grupo de socios de PH y en las AGEB más pobladas se incrementó la proporción para así asegurar no sólo representatividad en el ámbito municipal, sino para asegurar el número de socios de $\mathrm{PH}$, de acuerdo con la importancia de cada AGEB, lo cual elevó la muestra del grupo de control a 175. A continuación se muestran los resultados de las diferentes pruebas estadísticas.

\section{Resultados}

\subsection{Correlaciones}

Se prueba la asociación entre una variable binaria de pertenencia o no al programa $\mathrm{PH}$ y el nivel de las variables que constituyen los objetivos sociales de este programa. En el cuadro 2 se muestran los resultados al calcular los coeficientes de correlación bivariados, ahí se observa que sólo tres de las variables objetivo se asocian positivamente a la pertenencia a $\mathrm{PH}$.

\section{Cuadro 2}

Correlaciones entre las variables de comparación entre grupos y la pertenencia o no a $\mathrm{PH}$

\begin{tabular}{ll}
\hline \multicolumn{1}{c}{ Variable de posible contraste entre grupos } & \multicolumn{1}{c}{ Pertenencia o no a PH } \\
\hline Promedio de ingreso familiar & NES \\
Ahorro en pesos & NES \\
Ahorro como porcentaje del ingreso & NES \\
Nivel escolar del jefe del hogar & NES \\
Metros cuadrados de construcción & $0.129^{*}, \mathrm{n}=229$ \\
Número de cuartos en la vivienda & $0.230^{* *}, \mathrm{n}=228$ \\
Participación en grupos comunitarios & NES \\
Costo del proyecto de construcción & NES \\
Cuenta con un crédito actualmente & $0.136^{*}, \mathrm{n}=237$ \\
Nivel de felicidad & NES \\
\hline
\end{tabular}

NES: No estadísticamente significante. $n$ es el número de observaciones e incluye a ambos grupos, diferenciados mediante una variable binaria: pertenece a $\mathrm{PH}=1$, no pertenece $=0$.

**, * La correlación es significativa al nivel $\alpha=0.01$ y $\alpha=0.05$, respectivamente.

Fuente: Elaboración propia. 
Los coeficientes encontrados muestran que independientemente de los niveles de ingreso, ahorro, educación o número de personas en la vivienda, estar en PH se asocia positivamente con tener una vivienda más grande y con mayor número de cuartos. Por su parte, el porcentaje de ahorro de las familias depende del nivel de ingreso de la misma, sin importar si es o no socio de $\mathrm{PH}$, pues estar en el programa no se relaciona con ahorrar más. Con independencia del nivel de ingreso y educación del jefe de familia, los socios de PH usan más el crédito que los no socios. La participación social no está asociada con la pertenencia a PH y aquello que para los habitantes de Zinacantepec es la felicidad no está asociado con PH, ni con su nivel de ingreso, ni con el nivel educativo del jefe de familia, pero sí se relaciona de manera positiva con el tamaño de sus viviendas y de forma negativa con el número de consultas que hacen al médico por año.

\subsection{Diferencias entre medias y proporciones}

El análisis anterior permite observar algunas diferencias a favor del grupo de socios de $\mathrm{PH}$ en cuanto a algunas de las variables que constituyen objetivos sociales del programa. A continuación se hace un análisis comparativo de medias y proporciones de las variables de interés para ambos grupos en el estudio, con el fin de verificar si algunas de estas diferencias son estadísticamente significativas, los resultados obtenidos aparecen en el cuadro 3.

De acuerdo con los resultados presentados en el cuadro 3, se puede afirmar que en casi todas las variables, excepto en la de gasto en medicinas, la diferencia entre las medias aritméticas de las variables referentes al grupo de tratamiento y al de control son congruentes con lo esperado, y en general muestran condiciones más favorables para los socios de $\mathrm{PH}$, aun así, sólo algunas de estas diferencias son estadísticamente significativas. ${ }^{15}$ Se observa que si bien el nivel de ingreso entre los dos grupos no es diferente y tampoco lo es el número de habitantes por vivienda, el grupo de socios de PH cuenta con una vivienda más grande, tanto en metros cuadrados de construcción, como en número de cuartos, aun así, al observar el nivel de hacinamiento, medido por el cociente del número de personas con el número de cuartos por vivienda, éste es estadísticamente igual entre un grupo y otro. Por su parte, el material de construcción de las viviendas actualmente no es diferente entre los dos grupos. Es notorio

\footnotetext{
${ }^{15}$ En todas las comparaciones de medias se empleó también el procedimiento de análisis de varianza (ANOvA, por sus siglas en inglés) y se confirmaron todos los resultados, excepto el que relaciona el gasto en medicinas promedio anual por miembro de la vivienda con la participación o no en $\mathrm{PH}$, que bajo la prueba $t$ mostraba una diferencia significativa en el promedio de gasto, pero en la prueba del análisis de varianza no queda confirmada.
} 


\section{Cuadro 3}

\section{Prueba de diferencias de medias y proporciones para las variables de interés, entre el grupo de socios de $\mathrm{PH}$ y el de no socios}

\begin{tabular}{|c|c|c|c|c|}
\hline Variable & $\begin{array}{l}\text { Pertenencia } \\
\text { o no a PH }\end{array}$ & $n$ & $\begin{array}{c}\text { Media o } \\
\text { proporción }\end{array}$ & $\begin{array}{c}\text { ¿Existe o no } \\
\text { diferencia estadistica } \\
\text { entre las medias* } \\
\text { proporciones }^{* *} \text { de los } \\
\text { dos grupos? }\end{array}$ \\
\hline Ingreso promedio total & Sí & 62 & $\$ 3,600$ & \\
\hline $\begin{array}{l}\text { mensual de los integrantes } \\
\text { de la vivienda }\end{array}$ & No & 175 & $\$ 3,670$ & No \\
\hline Porcentaje de ahorro del & Sí & 62 & $3.11 \%$ & \\
\hline $\begin{array}{l}\text { ingreso total de los integran- } \\
\text { tes de la vivienda }\end{array}$ & No & 175 & $2.33 \%$ & No \\
\hline Proporción de jefes de hogar & Sí & 62 & 70.9 & \\
\hline $\begin{array}{l}\text { con educación hasta } \\
\text { secundaria completa }\end{array}$ & No & 175 & 87.4 & Sí \\
\hline Número de personas que & Sí & 62 & 6.39 & \\
\hline habitan la vivienda & No & 175 & 5.89 & No \\
\hline Tamaño de la vivienda en & Sí & 54 & $115.3 \mathrm{~m} 2$ & \\
\hline $\mathrm{m}^{2}$ de construcción & No & 175 & $99.86 \mathrm{~m} 2$ & Sí \\
\hline Número de cuartos en la & Sí & 53 & 6.22 & \\
\hline $\begin{array}{l}\text { vivienda (cocina, sala y } \\
\text { dormitorios) }\end{array}$ & No & 175 & 5.12 & Sí \\
\hline Techo con material & Sí & 54 & 99.2 & \\
\hline resistente $^{\mathrm{a}}(\%$ del total $)$ & No & 175 & 95.4 & No \\
\hline Paredes con material & Sí & 60 & 92.6 & \\
\hline resistente $^{\mathrm{b}}(\%$ del total $)$ & No & 175 & 89.1 & No \\
\hline Piso con material resistente ${ }^{c}$ & Sí & 61 & 98.4 & \\
\hline (\% del total) & No & 160 & 99.4 & No \\
\hline Visitas al médico promedio & Sí & 54 & 4.26 & \\
\hline $\begin{array}{l}\text { anual, por vivienda, por } \\
\text { enfermedades digestivas, de } \\
\text { la piel y pulmonares }\end{array}$ & No & 175 & 4.62 & No \\
\hline Visitas promedio al médico & Sí & 54 & 0.81 & \\
\hline $\begin{array}{l}\text { por ańo por ocupante de la } \\
\text { vivienda }\end{array}$ & No & 175 & 0.86 & No \\
\hline Gasto promedio anual en & Sí & 51 & $\$ 1,986.60$ & \\
\hline medicinas por vivienda ${ }^{\mathrm{d}}$ & No & 141 & $\$ 1,629.44$ & No \\
\hline Gasto promedio anual en & Sí & 51 & $\$ 356.20$ & \\
\hline $\begin{array}{l}\text { medicinas por ocupante de } \\
\text { la vivienda }\end{array}$ & No & 141 & $\$ 281.25$ & Sí \\
\hline Cuenta actualmente con & Sí & 62 & $11 \%$ & \\
\hline algún crédito & No & 175 & $4 \%$ & Sí \\
\hline Forma parte de algún grupo & Sí & 62 & $3 \%$ & \\
\hline comunitario & No & 175 & $2 \%$ & No \\
\hline Nivel de felicidad actual en & Sí & 62 & 8.47 & \\
\hline un rango del 0 a 10 & No & 175 & 8.37 & No \\
\hline
\end{tabular}

* Prueba $t$ de diferencia de medias entre dos grupos. Se empleó un nivel de confianza $\alpha=0.05$.

** Prueba $z$ de diferencia de proporciones entre dos grupos. Se empleó un nivel de confianza $\alpha=0.05$.

${ }^{a}$ Sólo se consideró material no resistente: madera, lámina y cartón.

${ }^{\mathrm{b}} \mathrm{A}$ lo anterior se agregó adobe.

' Se consideró losa, loseta, concreto y firme.

${ }^{\text {d }}$ Se eliminó a quienes reportan cero en su gasto en medicinas.

Fuente: Elaboración propia. 
también que el porcentaje de personas que ahora cuentan con un crédito es mayor en el grupo que fue socio de $\mathrm{PH}$. Por su parte, este grupo no parece estar ahorrando una mayor proporción de su ingreso que el de no socios, no consulta menos al médico, no está más integrado a grupos comunitarios y en general muestra un nivel igual de felicidad que los miembros del grupo que aún no han participado en PH. Si bien las diferencias entre las medias existen, y en su mayoría ilustrarían efectos de un mejor estado de los miembros de $\mathrm{PH}$ en lo referente a las variables de interés, las pruebas estadísticas todavía no permiten estimar que estas diferencias sean lo suficientemente amplias para estar seguros del efecto diferencial propiciado por el programa.

Con base en lo anterior, podemos decir que para los dos grupos de hogares que conforman el presente estudio, con características iguales de ingreso, ahorro, número de ocupantes, material de construcción en su vivienda (techo, paredes y piso), consultas al médico o gasto en medicamentos, el grupo que forma parte de PH logró consolidar una casa en promedio $15 \mathrm{~m}^{2}$ más grande y cuenta con un cuarto más que quienes no han entrado al programa. Esta diferencia significaría, en términos de la dinámica de crecimiento típico de la vivienda progresiva estimado por Bazant (2003), que los socios cuentan con un tamaño de vivienda que de no ser por su participación en $\mathrm{PH}$, hubieran alcanzado hasta cuatro años después. ${ }^{16}$

Este análisis puede ocultar posibles beneficios del programa PH. La situación actual de igualdad en muchas de las variables de interés, bien puede ser el resultado del cambio propiciado por $\mathrm{PH}$, mismo que habría permitido a sus socios alcanzar niveles de bienestar similares al de sus vecinos. Esta posibilidad nos llevaría a considerar el análisis de dobles diferencias dentro del grupo de socios. Como se comentó en la sección de metodología, este análisis no es tan factible en este estudio, ya que no se cuenta con la información de características socioeconómicas de base y comparativa entre los dos grupos bajo estudio, aun así, se considerarán algunos datos sobre las características de vivienda del municipio de Zinacantepec, provenientes del XII Censo de Población y Vivienda como un posible punto de contraste.

Al considerar los cambios directamente atribuibles al programa $\mathrm{PH}$ en el grupo de socios, las respuestas de los encuestados permiten ver los cambios ilustrados en el cuadro 4, que corresponden exclusivamente al aspecto de crecimiento patrimonial referente a la vivienda y que puede ser contrastado con los resultados del censo de población mencionado.

\footnotetext{
${ }^{16}$ De acuerdo con el estudio de Bazant (2003), referente a la vivienda progresiva en zonas de bajos ingresos del Estado de México, el avance trianual de la construcción en estas viviendas es de entre 10 y $12 \mathrm{~m}^{2}$. La equivalencia en este caso, para la diferencia de $15 \mathrm{~m}^{2}$, sería entre 4.5 y 3.75 ańos.
} 


\section{Cuadro 4}

\section{Prueba de diferencias de medias y proporciones para las variables de crecimiento y mejora de la vivienda, entre el grupo de socios de $\mathrm{PH}$, comparando situaciones al inicio y al final del programa}

\begin{tabular}{|c|c|c|c|c|}
\hline Variable de interés & $n$ & Antes & Después & $\begin{array}{c}\text { Pruebas t y z para } \\
\text { diferencias de medias* } \\
\text { y de proporciones** } \\
\text { ¿Difiere estadisticamente } \\
\text { la situación inicial de la } \\
\text { actual? }\end{array}$ \\
\hline $\begin{array}{l}\text { Porcentaje de viviendas con } \\
\text { techo de material resistente }\end{array}$ & 54 & 68.5 & 98.1 & Sí \\
\hline $\begin{array}{l}\text { Porcentaje de viviendas con } \\
\text { paredes de material resistente }\end{array}$ & 50 & 66.0 & 94.0 & Sí \\
\hline $\begin{array}{l}\text { Porcentaje de viviendas con } \\
\text { piso en material resistente }{ }^{c}\end{array}$ & 58 & 84.5 & 98.3 & Sí \\
\hline $\mathrm{m}^{2}$ promedio de construcción & 54 & 48.6 & 115.3 & Sí \\
\hline $\begin{array}{l}\text { Número promedio de cuartos } \\
\text { (cocina, sala y dormitorios) }\end{array}$ & 53 & 3.31 & 6.81 & Sí \\
\hline
\end{tabular}

* Prueba $t$ de diferencia de medias entre dos grupos. Se empleó un nivel de confianza $\alpha=0.05$.

** Prueba $z$ de diferencia de proporciones entre dos grupos. Se empleó un nivel de confianza $\alpha=$ 0.05 .

${ }^{a}$ Sólo se consideró material no resistente: madera, lámina y cartón.

${ }^{\mathrm{b}} \mathrm{A}$ lo anterior se agregó adobe.

' Se consideró losa, loseta, concreto y firme.

Fuente: Elaboración propia.

Como se puede observar, los resultados de mejora en calidad y tamaño de la vivienda son los esperados y favorables en la evaluación del programa. Por su parte, los porcentajes de techos, paredes y pisos de material resistente en las viviendas de los socios de $\mathrm{PH}$, según sus porcentajes antes de entrar al programa, son muy parecidos a los que se encontraron en general para el municipio de Zinacantepec en el año 2000, según cifras del XII Censo de Población y Vivienda. En este censo se reportó 68\% en techos, $68 \%$ en paredes y $76 \%$ en pisos. ${ }^{17}$ Los datos encontrados en el trabajo de campo para los dos grupos, hablarían de que el avance en provisión de material permanente en las viviendas de ambos grupos fue muy similar y no habría sido el grupo de PH el que más avanzó en este periodo. Una situación ligeramente diferente ocurrió con el promedio de número de cuartos, que de acuerdo con el censo fue de 3.6 por vivienda para Zinacantepec en $2000,{ }^{18}$ mientras que entre los socios de PH (en 2002-2003) fue de 3.1. En este caso podría decirse que el resultado confirma lo encon-

\footnotetext{
${ }^{17} \mathrm{http}: / /$ www.inegi.gob.mx/est/librerias/tabulados.asp?tabulado=tab_vi03a\&c=775\&e=, $7 \mathrm{de}$ noviembre de 2005.

${ }^{18} \mathrm{http}: / /$ www.inegi.gob.mx/est/librerias/tabulados.asp?tabulado=tab_vi07a\&c=780\&e=, $7 \mathrm{de}$ noviembre de 2005.
} 
trado al comparar la media de cuartos por grupo, así el número de cuartos fue una variable que creció más rápido entre los miembros de $\mathrm{PH}$ que entre el resto de la población en este municipio.

En cuanto al tamaño de la vivienda y el tiempo ahorrado en su avance, si hacemos nuevamente la comparación de lo logrado por los socios de PH versus la expectativa típica conformada por el grupo estudiado por Bazant (2003), referente al crecimiento promedio de la vivienda progresiva en el Estado de México, vemos que en el caso de estudio el crecimiento promedio observado de metros cuadrados de construcción equivalente a 66.7, normalmente llevaría a una familia de ingresos bajos un periodo de entre 16.5 y 19.8 años, mientras que a los socios de PH les llevó como máximo 4.5 años.

\subsection{Análisis de regresión}

Después de realizar las observaciones generales con base en la estadística descriptiva, y de explorar los posibles niveles de asociación entre la pertenencia o no a PH y la posible diferencia entre la condición que guardan algunas características del bienestar de los miembros de los grupos de tratamiento y control, la pregunta que se aborda ahora es: ¿cuál es el nivel de influencia de PH en el estado de las variables sociales objetivo de este programa, controlando para otras posibles variables concomitantes, que también podrían estar determinando el nivel de las variables sociales objetivo? Una importante diferencia entre este análisis y el anterior es que el presente permitirá medir el nivel de influencia, controlando simultáneamente para algunas posibles variables también determinantes de los niveles de las variables objetivo.

En el análisis de regresión se empleó la técnica de mínimos cuadrados ordinarios para explicar el comportamiento de las variables que representan objetivos sociales del programa. Cabe mencionar que aunque inicialmente se pensaba también controlar según la variable de género del jefe de la vivienda, esto no fue posible debido a que sólo en un caso una persona de género femenino fue identificada como jefa de la vivienda, lo cual no da suficientes observaciones para determinar diferencias.

No se encontraron modelos estadísticamente aceptables para explicar las variables de salud, ahorro, crédito, comunidad y felicidad. En todos los casos se incluyó la variable de pertenencia o no a PH como independiente, además en cada uno de los modelos correspondientes a cada una de las variables de interés se incluyeron otras variables explicativas. En el caso de salud se incorporaron como independientes el nivel de ingreso, el tamaño de las viviendas y el número de ocupantes por vivienda, pero no explicaron las variaciones de la dependiente. Al hacer pruebas para la variable de 
ahorro se consideró el ingreso, el gasto en salud, contar con crédito o tener expectativas en cuanto a éste, el ingreso siempre fue significativo y la pertenencia a PH también lo fue en algunos casos, pero el resto de las variables de prueba no lo fueron y en general los modelos encontrados no fueron estadísticamente significantes. En el caso del crédito, PH fue una variable significativa, pero el resto, entre las cuales se probaron el ingreso y salud no lo fueron, en este caso los modelos tampoco fueron estadísticamente aceptables. En cuanto al aspecto de lazos comunitarios no se encontraron variables explicativas, en realidad esta variable prácticamente es una constante, la participación comunitaria, como se señaló, es casi nula tanto en el grupo de tratamiento como en el de control. Por último, al modelar el tema de felicidad, el tamaño de la vivienda resultó ser la única variable determinante, pero dentro de modelos no estadísticamente significantes, donde también se consideró el ingreso.

Se encontraron resultados interesantes al modelar las variables del tamaño de la vivienda, tanto en el caso de metros cuadrados de construcción, como en la de número de cuartos, para la cual se estimaron modelos estadísticamente aceptables. En primera instancia se utilizó la técnica de mínimos cuadrados ordinarios, pero las ecuaciones estimadas mostraron heteroscedasticidad al emplear la prueba de White, por lo que para eliminar el problema se hicieron cálculos con mínimos cuadrados ponderados. A continuación se muestran las ecuaciones estimadas finales.

Tamaño de acuerdo con el total de metros cuadrados de construcción en la vivienda.

$$
\begin{aligned}
& \mathrm{m}^{2}=13.7144 \mathrm{PH}+0.3240 \mathrm{TT}+7.7918 \mathrm{NP}+\varepsilon \\
& \text { Prueba } t \text { : P-valores } \quad(0.0610) \quad(0.000) \quad(0.000) \\
& \text { FIV } \\
& \text { (1.012) (1.001) }
\end{aligned}
$$

Prueba de Kolmogorov-Smirnov de normalidad del error: $\mathrm{p}$-valor $=0.000$

Tamaño de acuerdo con el número de cuartos en la vivienda.

$$
\begin{aligned}
& \mathrm{NC}=4.3044+1.0211 \mathrm{PH}+0.1370 \mathrm{NP}+\varepsilon \\
& \begin{array}{l}
\text { Prueba } t: \text { P-valores } \\
\text { FIV }
\end{array} \\
& \mathrm{R}_{\text {aj }}^{2}=0.1918 \\
& \mathrm{DW}=1.7272 \\
& \text { Prueba de Kolmogorov-Smirnov de normalidad del error: } \mathrm{p} \text {-valor }=0.000
\end{aligned}
$$


donde $\mathrm{m}^{2}$ es el número de metros cuadrados de construcción en la vivienda; NC es el número de cuartos (incluye cocina, sala y dormitorios); $\mathrm{PH}$ es binaria de pertenencia $=1$ o no $=0$ a Patrimonio Hoy; тт es el tamaño en $\mathrm{m}^{2}$ del terreno donde está edificada la vivienda, y NP es el número de ocupantes.

En general ambas ecuaciones son estadísticamente significantes (prueba $\mathrm{F}$ del modelo) y en cada uno de los coeficientes (prueba $t$ de student), no muestran problemas de multicolinealidad (verificado mediante los factores incrementales de varianza, FIV), autocorrelación (empleando el estadístico Durbin Watson, Dw), normalidad del error (mediante la prueba de Kolmogorov-Smirnov, también se empleó Jarque-Vera y los resultados fueron los mismos) y varianza constante del término del error por observación (se estimó con mínimos cuadrados ponderados). Nuevamente se confirman los resultados que se obtuvieron en las pruebas iniciales, ser socio de PH incide positivamente en el tamaño de la vivienda según metros cuadrados de construcción y número de cuartos.

En resumen, se puede decir que el análisis realizado por medio de las diferentes pruebas estadísticas confirma el efecto positivo que el programa PH ha tenido en el tamaño y calidad de las viviendas en los socios de Zinacantepec, así como en el menor tiempo en que éstos alcanzaron dichos tamaños de construcción. Por su parte, este estudio no permite confirmar que esta mejora en la vivienda y/o la participación en el programa hayan conducido al socio a consultar menos al médico, a aumentar su ahorro y a participar más en la comunidad. Adicionalmente, llega a resultados que si bien no permiten descartar la influencia de $\mathrm{PH}$, tampoco permiten asegurar que ésta existe en lo tocante a un mayor uso del crédito comercial y/o bancario por parte del socio después de participar en el programa.

También se hizo una aproximación de la rentabilidad obtenida al participar en $\mathrm{PH}$, se estimó que el costo de construcción de un $\mathrm{m}^{2}$ considerando materiales y mano de obra -en el caso de autoconstrucción también se incluyó un pago por mano de obra- no fue distinto entre los dos grupos bajo comparación, alcanzando un promedio de 513 pesos; por su parte, el precio promedio de venta por $\mathrm{m}^{2}$ en Zinacantepec es de 2,000 pesos. Así, la creación de riqueza patrimonial se confirma como lo han sugerido otros autores (Herbst, 2002; Sandoval 2005a) y, en este caso, es ampliamente rentable.

\section{Conclusiones y recomendaciones}

En vista de los grandes déficits habitacionales que prevalecen en México, ya sean medidos por el número de cuartos faltantes, las condiciones precarias de millones de viviendas y/o la falta de ellas, y ante las fuertes limi- 
taciones que más de 70\% de las familias mexicanas enfrentan para acceder a un crédito que les permita construir una mejor vivienda, el presente estudio encuentra al programa Patrimonio Hoy, de Cemex, como una alternativa de financiamiento, que ha permitido a las familias de ingreso bajo, habitantes del municipio de Zinacantepec, Estado de México, construir una vivienda más grande, de mejor calidad y en menor tiempo, que la que hubieran podido edificar si este programa no existiera.

Como otros autores ya lo han ilustrado, PH representa para la empresa Cemex una importante oportunidad de mercado. Los análisis realizados en este estudio comprueban, además, que esta iniciativa, en la cual se instrumenta una estrategia novedosa para llegar a mercados poco atendidos, es un medio que permite a las familias de bajos ingresos reducir su nivel de pobreza patrimonial en menor tiempo e implementar proyectos de construcción que les son ampliamente rentables.

Si bien es posible afirmar que el programa está alcanzando algunos de los objetivos sociales directos que se ha trazado, consistentes en permitir a sus socios contar con una vivienda mejor construida y más grande en menor tiempo y a menor costo, por otro lado, los resultados estadísticos no permiten decir lo mismo del logro de otros de los objetivos sociales que también se propone: crear en el socio el hábito del ahorro, mejorar su estado de salud, integrarlo más socialmente, darle una experiencia de crédito que le lleve a usar más otro tipo de instrumentos crediticios y aumentar su nivel de bienestar general. Quizá falta esperar algunos años más para ver reflejado el efecto de aumento en patrimonio habitacional sobre estos otros objetivos. Por su parte, los resultados bien pueden reflejar la necesidad de complementar la estrategia actual con medidas más directas y de mayor alcance, utilizando incentivos ${ }^{19}$ más idóneos, que fomenten un crecimiento sustentable en la ejecución del programa para hacerlo incidir en estos otros objetivos sociales que eventualmente redunden en una mejor calidad de vida.

Los niveles de rentabilidad social ${ }^{20}$ encontrados, cercanos a $290 \%$, así como la confirmación de la relación positiva entre la participación en $\mathrm{PH}$ y el tamaño y condiciones de la vivienda, dan pautas importantes para que la empresa pudiera incluso buscar el apoyo de los gobiernos para extender este proyecto, con la justificación de los beneficios sociales netos

${ }^{19}$ El presente trabajo no se detuvo a estudiar el por qué del no cumplimiento de cierta parte de los objetivos de $\mathrm{PH}$, por lo que se pudo observar en la operación del programa, los incentivos al personal están inclinados únicamente a captar más socios, no a reconocer el cambio en variables que hablen sobre los diversos aspectos del bienestar que PH busca mejorar en aquellas familias que se asocian al programa.

${ }^{20}$ En este caso, se usó el término rentabilidad social para referir el rendimiento sobre la inversión financiera realizada por el beneficiario de $\mathrm{PH}$, y no confundirla con la rentabilidad propia de la empresa que administra el programa. 
que ha mostrado. En 2005 el programa había alcanzado más de 90,000 socios en toda la República Mexicana, los resultados para Zinacantepec ilustran que la participación en el programa resulta en un diferencia de $13.68 \mathrm{~m}^{2}$ en promedio como efecto del programa, cada uno de los cuales reporta al socio un aumento patrimonial de $1,486.68^{21}$ pesos, es decir un total de 20,337.78 pesos, si esto se extiende a los 90,000 socios registrados al año 2005, se hablaría de la creación de una riqueza patrimonial en el ámbito de poco más de 183 millones de pesos, sin comprometer ningún recurso público. En términos del número de cuartos, los resultados para Zinacantepec ilustran que la participación en PH significa para el socio promedio contar con un cuarto más que quien no participa, extrapolando el resultado al ámbito nacional se diría que si los promedios de permanencia en el programa fueran similares a los encontrados en Zinacantepec, para el resto de la República el déficit habitacional se habría reducido en 90,000 cuartos, lo cual representa apenas $1.1 \%$ del déficit habitacional medido en número de cuartos, según cifras del censo del año 2000. Esto refiere la amplia necesidad y oportunidad por llevar a cabo acciones que permitan la extensión del programa a un mayor número de socios, e incluso estudiar su viabilidad no sólo en zonas urbanas, sino también a zonas rurales.

Finalmente, el rápido crecimiento del programa a lo largo de las zonas urbanas de ingreso bajo en México, aunado a las buenas perspectivas de mercado que PH representa para Cemex y la alternativa que significa para sus usuarios, quienes de esta forma se están haciendo más rápido de un mejor y mayor patrimonio, viene a conformar la imagen de una importante estrategia de responsabilidad social, que muy probablemente seguirá rindiendo frutos por largo tiempo, tanto para la empresa como para la sociedad, para la cual se contribuye de manera directa en la reducción del problema de la pobreza patrimonial y sirve como una alternativa financiera de construcción de vivienda para grupos donde el sistema financiero formal aún no es accesible.

Quedan interesantes puntos a estudiar en trabajos subsecuentes, pues como se dijo al principio, este trabajo versa sobre lo que ha sucedido en uno de los municipios, pero sería interesante compararlo con resultados de estudios similares en otros municipios. Por su parte, la evaluación de efectos comunitarios podría ser más del interés de los gobiernos. Por último, sería importante trabajar en el diseño de contratos de incentivos

\footnotetext{
${ }^{21}$ Se considera la diferencia entre el costo promedio de construcción con $\mathrm{PH}$ (incluido un pago por mano de obra en caso de autoconstrucción) de $513.32 \mathrm{~m}^{2}$ y un valor de mercado promedio del mismo en la zona de Zincantepec de 2,000 pesos. El cálculo puede variar de un lugar a otro del país, donde si bien el costo de los materiales es muy similar, el costo de mano de obra y el valor de mercado pueden diferir ampliamente.
} 
óptimos, que consideren las acciones del personal de PH para lograr algunos de los objetivos sociales que sigue el programa y que hasta ahora no ha alcanzado.

\section{Bibliografía}

Aguilar, María y Ezequiel Ander-Egg (1992), Evaluación de servicios y programas sociales, Siglo XXI, Madrid.

Banco Mundial (2002a), Informe sobre el desarrollo mundial, 2003, Banco Mundial, Washington.

Banco Mundial (2002b), Informe sobre el desarrollo mundial, 2003: Panorama general, Banco Mundial, Washington.

Banco Mundial (2004), La pobreza en México: una evaluación de las condiciones, las tendencias y la estrategia del gobierno, Banco Mundial, México.

Bazant, Jan (2003), Viviendas progresivas: construcción de vivienda por familias de bajos ingresos, Trillas, México.

Burke, Lee y Jeanne M. Logsdon (1996), "How corporate social responsibility pays off”, Long Range Planning, 29 (4), Elsevier Science, Amsterdam, pp. 495-502.

Cacho, Yalín (2005), "Portazo hipotecario a familias de bajos ingresos", El Financiero, 26 de julio, México.

Campos, Pilar (2005), El ahorro popular en México: acumulando activos para superar la pobreza, Porrúa-Cidac, México.

CIDAC-SHF (Centro de Investigación y Documentación de la Casa, A.C.Sociedad Hipotecaria Federal) (2009), Estado actual de la vivienda en México 2009, CIDAC-SHF, México.

Coldewey, Chris (2005), “вор in action: Cemex’s Patrimonio Hoy: Triggers for innovation -New models for change and social entrepreneurship", World Changing, <http:// www.worldchanging. com/archives/002949.html>, 26 de agosto de 2005. 
Coneval (Consejo Nacional de Evaluación de la Política de Desarrollo Social), Medición de la pobreza multidimensional, <http:// www. coneval.gob.mx/coneval2/htmls/medicion_pobreza/HomeMedicionPobreza.jsp?categorias=MED_POBREZA,MED_POBREZA-med_multi>, 17 de mayo de 2010.

Correa, María, Sharon Flynn y Alon Amit (2004), Responsabilidad social corporativa en América Latina: una visión empresarial, CEPAL, Santiago de Chile.

Cunningham, Katie y Marc Ricks (2004), "Why measure. Nonprofits use metrics to show that they are efficient. But what if donors don't care, Stanford Social Innovation Review", <http:// www. ssireview.com/pdf/2004SU_feature_cunningham.pdf?>, 19 de octubre de 2010.

Damián, Araceli (2005), "Entre el desastre, la corrupción y la pobreza”, El Financiero, 10 de octubre, México.

"Dan a Cemex premio Hábitat" (2009), El Norte, 5 de octubre, <http:// www.elnorte.com/negocios/articulo/519/1036501/default.asp>.

DeTombe, Dorien (2003), "Handling complex societal problems", en Henk Becker y Frank Vanclay (eds.), The international handbook of social impact assessment: Conceptual and methodological advances, Edward Elgar, Massachusetts, pp. 278-295.

Flores, María (2004), "For profit development: Good business, good for all”, <http:// weblog.cheskin.net/blog/archives/000256.html>, $1^{\circ}$ de noviembre de 2005.

Freeman, Daniel (1987), Applied categorical data analysis, Marcel Dekker, Nueva York.

González, José (2003), "Estado, política social de vivienda y autoconstrucción: el sistema de consolidación habitacional en las urbanizaciones populares bajo el neoliberalismo (El caso del área metropolitana de Monterrey)", tesis doctoral, Universidad Autónoma de Nuevo León, México.

Herbst, Kris (2002), "Brindándole la posibilidad a los pobres de construir su propia casa: ganancias y desarrollo social", Changemakers.net 
Journal, <http:// www.changemakers.net/journal/02september/ herbstep.cfm>, 19 de octubre de 2010.

Husted, Bryan y José Salazar (2005), "Un estudio exploratorio sobre la estrategia social de empresas grandes ubicadas en México", Contaduría y Administración, 215, Universidad Autónoma del Estado de México, Toluca, pp. 9-23.

Husted, Bryan y José Salazar (2006), “Taking Friedman seriously: Maximazing profits and social performance ", Journal of Management Studies, 43 (1), Wiley-Blackwell, Oxford, pp. 75-91.

Iracheta, Alfonso (2001), México: Estudio sobre la vivienda de bajo ingreso. Limitaciones en la oferta de vivienda, Banco Mundial, Washington.

Kunz-Bolaños, Ignacio e Irma Romero Vadillo (2008), "Naturaleza y dimensión del rezago habitacional en México", Economía, Sociedad y Territorio, 8 (26), El Colegio Mexiquense, Zinacantepec, pp. 415-449.

Lara, María (2000), Filantropía empresarial: convicción y estrategia, Pax, México.

Levin, Richard (1981), Statistics for management, Prentice-Hall, Nueva Jersey.

Peinado, Estrella (2004), Corporate social responsibility in Latin America and the Caribbean, Banco Interamericano de Desarrollo, Washington.

Rodríguez, Javier (2005), "Sin vivienda, más de 11 millones de personas", El Financiero, 5 de octubre, México.

Salazar, Sabrina (2004), "La responsabilidad social corporativa en México como una herramienta estratégica para la competitividad: un enfoque econométrico y prospectivo", tesis de maestría, Instituto Tecnológico y de Estudios Superiores de Monterrey, México.

Sandoval, Ricardo (2005a), "Block by block: How one of the world's largest companies builds loyalty among Mexico's poor", Stanford Social Innovation Review, <http:// www.ssireview.com/pdf/2005 SU_feature_sandoval.pdf $>, 19$ de octubre de 2010. 
Sandoval, Ricardo (2005b), "Lo pequeño es hermoso (Small is beautiful) Banks in Mexico say that micro loans are helping the poor while boosting their profits", Stanford Social Innovation Review, <http:// www.ssireview.com/articles/index.php?browseDate= Fall+2005>, 19 de octubre de 2010.

Sedesol (Secretaría de Desarrollo Social) (2003), Evaluación de resultados del programa de educación, salud y alimentación. Metodología de la evaluación de Progresa 2000, Sedesol, México.

Recibido: 6 de octubre de 2009. Aceptado: 20 de mayo de 2010.

José de Jesús Salazar-Cantú. Es doctor por el Instituto de Investigaciones Sociales de la Universidad Autónoma de Nuevo León (UANL); realizó estudios de licenciatura en economía y maestría en ciencias de la administración en el Instituto Tecnológico y Estudios Superiores de Monterrey (ITESM) y de maestría en economía en la UANL. Actualmente es investigador nivel I en el Sistema Nacional de Investigadores (SNI); es profesor titular en el Departamento de Economía del ITESM, campus Monterrey; es miembro de la cátedra de desarrollo económico y social del ITESM y editor de la Revista Estudiantil de Economía. Sus líneas de investigación actuales son: responsabilidad social corporativa, evaluación de programas sociales y economía internacional. Entre sus publicaciones más recientes se encuentran: "Principals and agents: Further thoughts on the friedmanite critique of corporate social responsibility", en The Oxford Handbook of Corporate Social Responsibility, Oxford University Press, Oxford, pp. 137-155 (2008); "Responsabilidad social de las empresas y beneficios privados: el impacto de la orientación estratégica en empresas grandes ubicadas en México", Economía, Sociedad y Territorio, 27, El Colegio Mexiquense, Zinacantepec, pp. $739-768$ (2008); "Les effets des exportations sur la croissance du produit manufacturier dans les Etats mexicain", en F. Pinot (ed.), La mondialisation et ses effets: nouvelles approches, nouveaux débats, L’Harmattan, París, pp. 71-80 (2006).

Bryan W. Husted. Es doctor por la Universidad de California, Berkeley. Actualmente ocupa la cátedra Erivan K. Haub en negocios y sustentabilidad; es director del Centro de Excelencia en Negocios Responsables, de la Universidad de York, en Toronto. Es miembro del consejo editorial de Business Ethics Quarterly, Journal of Management Studies y Long Range Planning. Sus líneas de investigación abordan la responsabilidad social de 
la empresa y el desarrollo sustentable. Entre sus publicaciones más recientes se encuentran: "Principals and agents: Further thoughts on the friedmanite critique of corporate social responsibility", en The Oxford Handbook of Corporate Social Responsibility, Oxford University Press, Oxford, pp. 137-155 (2008); "Agency, information, and the structure of moral problems in business", Organization Studies, 28 (2), Sage, Londres, pp. 177-195 (2007); "Corporate social strategy in multinational enterprises: Antecedents and value creation", Journal of Business Ethics, Springer, Dordrecht, 74 (4), pp. 177-195 (2007).

Esthela Gutiérrez-Garza. Es doctora por la Universidad de París viII. Actualmente es investigadora nivel III en el Sistema Nacional de Investigadores (SNI); es miembro de la Academia Mexicana de Ciencias, secretaria de la Secretaría de Desarrollo Sustentable de la Universidad Autónoma de Nuevo León (UANL) y de los programas de maestría y doctorado en ciencias sociales con orientación en desarrollo sustentable del Instituto de Investigaciones Sociales de la UANL; es directora de la revista Trayectorias de la UANL. Sus líneas de investigación son: teorías del desarrollo, desarrollo sustentable, relaciones industriales, Estado y política social y bienestar social. Entre sus publicaciones destacan: Escenarios de sustentabilidad industrial: Nuevo León 1988-2004, Plaza y Valdés-uAnl (2009); De las teorías del desarrollo al desarrollo sustentable: una historia multidisciplinaria, Siglo XXI-UANL (2010); México: democracia, participación social y proyecto de nación, Siglo XXI-UANL (2009).

Karim Acuña-Askar. Es doctor por la Universidad de Tulane. Actualmente es profesor de tiempo completo en el Departamento de Microbiología de la UANL. Es investigador nivel I del Sistema Nacional de Investigadores (sNI); miembro de la cátedra sobre ambiente y sustentabilidad de la UANL, y pertenece a las siguientes asociaciones internacionales: U.S. Water Environment Federation, International Water Association, American Chemical Society, American Society for Microbiology, New York Academy of Sciences y Sociedad Química de México. Sus líneas actuales de investigación son: tratamiento y calidad del agua, monitoreo de microorganismos patógenos de importancia ambiental, y diseño de programas tecnológicos de sustentabilidad ambiental. Entre sus publicaciones se encuentran: " $\mathrm{Ci}$ nética de biodegradación de mezclas de втеох-емтв por medio de un proceso de bioaumentación”, Ciencia UANL, 11 (1), UANL, Monterrey, pp. 63-68 (2008); "Mineral nutrition requirements for Bermuda grass grown on a professional soccer field, Acta Hort. (ISHS), 843, pp. 129-136 (2009); "Growth mineral uptake and stem elongation of Lillium spp. As a function of plant density”, Acta Hort. (ISHS), 843, pp. 81-88 (2009). 